\title{
Explicit Solutions of the Boundary Value Problems for an Ellipse with Double Porosity
}

\author{
Lamara Bitsadze and Natela Zirakashvili \\ Ilia Vekua Institute of Applied Mathematics, Ivane Javakhishvili Tbilisi State University, 2 University Street, 0186 Tbilisi, Georgia \\ Correspondence should be addressed to Natela Zirakashvili; natzira@yahoo.com
}

Received 18 March 2016; Revised 26 June 2016; Accepted 4 July 2016

Academic Editor: Xiao-Jun Yang

Copyright ( 2016 L. Bitsadze and N. Zirakashvili. This is an open access article distributed under the Creative Commons Attribution License, which permits unrestricted use, distribution, and reproduction in any medium, provided the original work is properly cited.

\begin{abstract}
The basic two-dimensional boundary value problems of the fully coupled linear equilibrium theory of elasticity for solids with double porosity structure are reduced to the solvability of two types of a problem. The first one is the BVPs for the equations of classical elasticity of isotropic bodies, and the other is the BVPs for the equations of pore and fissure fluid pressures. The solutions of these equations are presented by means of elementary (harmonic, metaharmonic, and biharmonic) functions. On the basis of the gained results, we constructed an explicit solution of some basic BVPs for an ellipse in the form of absolutely uniformly convergent series.
\end{abstract}

\section{Introduction}

In a material with two degrees of porosity, there are two pore systems, the primary and the secondary. For example, in a fissured rock (i.e., a mass of porous blocks separated from one another with an interconnected and continuously distributed system of fissures), most of the porosity is provided by the pores of the blocks or primary porosity, while most of permeability is provided by the fissures or secondary porosity. When fluid flow and deformation processes occur simultaneously, three coupled partial differential equations can be derived $[1,2]$ to describe the relationships governing pressure in the primary and secondary pores (and therefore the mass exchange between them) and the displacement of the solid.

A theory of consolidation with double porosity structure was proposed by Wilson and Aifantis [1]. The physical and mathematical foundations of the theory of double porosity were considered in the papers [1-3], where analytical solutions of the relevant equations are also given. This theory unifies a model proposed by Biot for the consolidation of deformable single porosity media with a model proposed by Barenblatt for seepage in indeformable media with two degrees of porosity. The basic results and the historical information on the theory of porous media were summarized by
De Boer [4]. However, Aifantis' quasi-static theory ignored the cross-coupling effect between the volume change of the pores and fissures in the system. The cross-coupled terms were included in the equations of conservation of mass for the pore and fissure fluid and in Darcy's law for solids with double porosity structure by several authors [5-8].

Porous media theories play an important role in many branches of engineering, including material science, petroleum industry, chemical engineering, and soil mechanics, as well as biomechanics. In recent years, many authors investigated the BVPs of the 2D and 3D theories of elasticity for materials with double porosity structure, publishing a large number of papers (some of these results can be seen in [9-17] and references therein). In those works, the explicit solutions on some BVPs in the form of series are given in a form useful for the engineering practice.

In the present paper, the basic two-dimensional boundary value problems of the fully coupled linear equilibrium theory of elasticity for solids with double porosity structure are reduced to the solvability of two types of a problem. The first one is similar to the BVPs for the equations of classical elasticity of isotropic bodies, while the second one is the BVPs for the equations of the pore and fissure fluid pressures. The solutions of these equations are presented by means of elementary (harmonic, metaharmonic, and biharmonic) functions. On 
the basis of the gained results, we constructed an explicit solution of some basic BVPs for an ellipse in the form of absolutely uniformly convergent series.

\section{Basic Equations}

Let $x=\left(x_{1}, x_{2}\right)$ be a point of the Euclidean $2 \mathrm{D}$ space $R^{2}$. In what follows, we consider an ellipse with a double porosity structure that occupies the region $\Omega$ of $R^{2}$.

The system of homogeneous equations of the linear equilibrium theory of elasticity for solids with double porosity structure can be written as follows [9]:

$$
\begin{aligned}
\mu \Delta \mathbf{u}+(\lambda+\mu) \operatorname{grad} \operatorname{div} \mathbf{u} & =\operatorname{grad}\left(\beta_{1} p_{1}+\beta_{2} p_{2}\right) \\
\left(k_{1} \Delta-\gamma\right) p_{1}+\left(k_{12} \Delta+\gamma\right) p_{2} & =0, \\
\left(k_{21} \Delta+\gamma\right) p_{1}+\left(k_{2} \Delta-\gamma\right) p_{2} & =0,
\end{aligned}
$$

where $\mathbf{u}=\left(u_{1}(\mathbf{x}), u_{2}(\mathbf{x})\right)^{T}$ is the displacement vector in a solid, $p_{1}(\mathbf{x})$ and $p_{2}(\mathbf{x})$ are the pore and fissure fluid pressures, respectively, $\beta_{1}$ and $\beta_{2}$ are the effective stress parameters, $\gamma>0$ is the internal transport coefficient and corresponds to the fluid transfer rate with respect to the intensity of flow between the pores and fissures, $\lambda, \mu, k_{1}, k_{2}$ are all constitutive coefficients, $k_{j}=\kappa_{j} / \mu^{\prime}(j=1,2), k_{12}=\kappa_{12} / \mu^{\prime}, k_{21}=\kappa_{21} / \mu^{\prime}$, $\mu^{\prime}$ is the fluid viscosity, $\kappa_{1}$ and $\kappa_{2}$ are the macroscopic intrinsic permeabilities associated with matrix and fissure porosity, respectively, $\kappa_{12}$ and $\kappa_{21}$ are the cross-coupling permeabilities for fluid flow at the interface between the matrix and fissure phases, and $\Delta$ is the two-dimensional Laplace operator. We consider the vectors as column matrices, if necessary. Throughout this paper, it is assumed that $\beta_{1}^{2}+\beta_{2}^{2}>0$. Superscript " $T$ " denotes transposition. We will suppose that

$$
\begin{aligned}
k_{1} & >0, \\
k_{2} & >0, \\
k_{1} k_{2}-k_{12} k_{21} & >0, \\
k_{0} & =k_{1}+k_{2}+k_{12}+k_{21}>0 .
\end{aligned}
$$

Note that BVPs for system (2) which contain $p_{1}(\mathbf{x})$ and $p_{2}(\mathbf{x})$ can be investigated separately. Then if supposing $p_{j}(\mathbf{x})$ as known, we can study BVPs for system (1) with respect to $\mathbf{u}(\mathbf{x})$. By combining the obtained results, we arrive at explicit solutions of BVPs for systems (1)-(2).

Obviously, from system (2), we have the following equations for $p_{1}(\mathbf{x})$ and $p_{2}(\mathbf{x})$ :

$$
\Delta\left(\Delta+\lambda_{1}^{2}\right) p_{j}=0, \quad j=1,2,
$$

where

$$
\lambda_{1}=i \sqrt{\frac{\gamma k_{0}}{k_{1} k_{2}-k_{12} k_{21}}}, \quad k_{0}=k_{1}+k_{2}+k_{12}+k_{21} .
$$
form

It is easy to see that the solutions of system (2) have the

$$
\begin{aligned}
& p_{1}=\phi+m_{1} \phi_{1}, \\
& p_{2}=\phi+\phi_{1},
\end{aligned}
$$

where

$$
\begin{aligned}
m_{1} & =-\frac{k_{2}+k_{12}}{k_{1}+k_{21}}, \\
\Delta \phi & =0 \\
\left(\Delta+\lambda_{1}^{2}\right) \phi_{1} & =0 .
\end{aligned}
$$

First, we assume $p_{j}$ to be known. Then, for $\mathbf{u}(\mathbf{x})$, we get the following nonhomogeneous equation:

$$
\begin{aligned}
\mu \Delta & \mathbf{u}+(\lambda+\mu) \operatorname{grad} \operatorname{div} \mathbf{u} \\
& =\operatorname{grad}\left[\left(\beta_{1}+\beta_{2}\right) \phi+\left(\beta_{1} m_{1}+\beta_{2}\right) \phi_{1}\right] .
\end{aligned}
$$

It is well known that the general solution of (8) has the form

$$
\mathbf{u}=\mathbf{v}+\mathbf{v}_{\mathbf{0}},
$$

where $\mathbf{v}$ is a general solution of the equation

$$
\mu \Delta \mathbf{v}+(\lambda+\mu) \operatorname{grad} \operatorname{div} \mathbf{v}=0
$$

and $\mathbf{v}_{\mathbf{0}}$ is a particular solution of the nonhomogeneous equation

$$
\mathbf{v}_{\mathbf{0}}=\frac{1}{\lambda+2 \mu} \operatorname{grad}\left[\left(\beta_{1}+\beta_{2}\right) \varphi_{0}-\frac{\beta_{1} m_{1}+\beta_{2}}{\lambda_{1}^{2}} \phi_{1}\right] ;
$$

here,

$$
\begin{aligned}
\Delta \phi & =0, \\
\Delta \varphi_{0} & =\phi, \\
\left(\Delta+\lambda_{1}^{2}\right) \phi_{1} & =0 .
\end{aligned}
$$

\section{Basic Equations in the Elliptic Coordinate System}

Let us rewrite equalities (6), (9), and (10)-(12) in elliptic coordinates system $\xi, \eta(0 \leq \xi<\infty, 0 \leq \eta<2 \pi)$ (see Appendix A). We obtain the following.

Formula (9) takes the form

$$
\widetilde{\mathbf{u}}=\widetilde{\mathbf{v}}+\widetilde{\mathbf{v}}_{\mathbf{0}} .
$$

Formula (11) takes the form

$$
\widetilde{\mathbf{v}}_{\mathbf{0}}=\frac{1}{\lambda+2 \mu} \frac{1}{h}\left(\frac{\partial \Psi}{\partial \xi} \vec{e}_{\xi}+\frac{\partial \Psi}{\partial \eta} \vec{e}_{\eta}\right),
$$

where

$$
\begin{aligned}
\Psi & :=\left(\beta_{1}+\beta_{2}\right) \bar{\varphi}_{0}-\frac{\beta_{1} m_{1}+\beta_{2}}{\lambda_{1}^{2}} \overline{\phi_{1}}, \\
h & =\sqrt{\sinh ^{2} \xi+\sin ^{2} \eta} \\
\overline{\varphi_{0}} & =\overline{\varphi_{0}}(\xi, \eta), \\
\overline{\phi_{1}} & =\overline{\phi_{1}}(\xi, \eta) .
\end{aligned}
$$


Equations (12) take the form

$$
\begin{aligned}
\Delta \bar{\phi}(\xi, \eta) & =0 \\
\frac{1}{h^{2}}\left(\frac{\partial^{2} \overline{\phi_{1}}}{\partial \xi^{2}}+\frac{\partial^{2} \overline{\phi_{1}}}{\partial \eta^{2}}\right)+\lambda_{1}^{2} \overline{\phi_{1}} & =0 \\
\frac{1}{h^{2}}\left(\frac{\partial^{2} \bar{\varphi}_{0}}{\partial \xi^{2}}+\frac{\partial^{2} \bar{\varphi}_{0}}{\partial \eta^{2}}\right) & =\bar{\phi}
\end{aligned}
$$

Expressions (6) can be rewritten in the following form:

$$
\begin{aligned}
& \bar{p}_{1}=\bar{\phi}+m_{1} \bar{\phi}_{1}, \\
& \bar{p}_{2}=\bar{\phi}+\bar{\phi}_{1} .
\end{aligned}
$$

Equation (10) in the elliptic coordinates system takes the form [18-21]

$$
\begin{aligned}
& \frac{\partial D}{\partial \xi}-\frac{\partial K}{\partial \eta}=0, \\
& \frac{\partial \bar{u}}{\partial \xi}+\frac{\partial \bar{v}}{\partial \eta}=\frac{\kappa-2}{\kappa \mu^{\prime}} h_{0}^{2} D, \\
& \frac{\partial D}{\partial \eta}+\frac{\partial K}{\partial \xi}=0, \\
& \frac{\partial \bar{v}}{\partial \xi}-\frac{\partial \bar{u}}{\partial \eta}=\frac{1}{\mu^{\prime}} h_{0}^{2} K,
\end{aligned}
$$

where $\kappa=4(1-\nu), \mu=E / 2(1-\nu), h_{0}:=$ $\sqrt{\cosh (2 \xi)-\cos (2 \eta)}, \bar{u}=2 h \widetilde{v}_{1} / c^{2}, \bar{v}=2 h \widetilde{v}_{2} / c^{2}$, (in what follows, we assume that $c=1), \widetilde{v}_{1}$ and $\widetilde{v}_{2}$ are the components of displacement vector $\widetilde{\mathbf{v}}\left(\widetilde{v}_{1}, \widetilde{v}_{2}\right)$ along the normal and the tangent to the curve $\xi=$ const, $((\kappa-2) / \kappa \mu) D$ is divergence of displacement vector $\widetilde{\mathbf{v}},(1 / \mu) K$ is a rotor of displacement vector $\widetilde{\mathbf{v}}, v$ is Poisson's ratio, and $E$ is Young's modulus.

Hooke's law can be written as follows:

$$
\begin{aligned}
& \frac{h_{0}^{2}}{\mu} \sigma_{\xi \xi}=\frac{h_{0}^{2}}{\mu} D-2 \frac{\partial \bar{v}}{\partial \eta}-\frac{2}{h_{0}^{2}}[\sinh (2 \xi) \bar{u}-\sin (2 \eta) \bar{v}], \\
& \frac{h_{0}^{2}}{\mu} \sigma_{\eta \eta}=\frac{h_{0}^{2}}{\mu} D-2 \frac{\partial \bar{u}}{\partial \xi}+\frac{2}{h_{0}^{2}}[\sinh (2 \xi) \bar{u}-\sin (2 \eta) \bar{v}] \\
& \frac{h_{0}^{2}}{\mu} \tau_{\xi \eta}=\frac{h_{0}^{2}}{\mu} K+2 \frac{\partial \bar{u}}{\partial \eta}-\frac{2}{h_{0}^{2}}[\sin (2 \eta) \bar{u}+\sinh (2 \xi) \bar{v}]
\end{aligned}
$$

\section{Boundary Value Problems}

For systems (1) and (2), we pose the following BVPs.

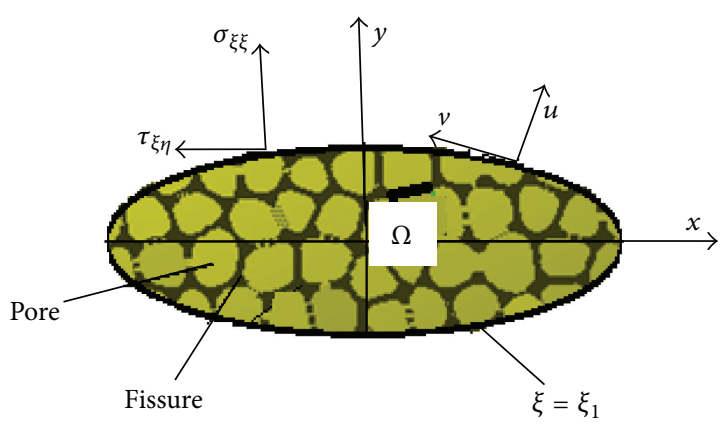

FIGURE 1: Ellipse with double porosity.

Find in domain $\Omega=\left\{0 \leq \xi<\xi_{1}, 0 \leq \eta<2 \pi\right\}$ a regular solution $\mathbf{U}\left(\mathbf{u}, p_{1}, p_{2}\right) \in C^{2}(\Omega)$ to systems (1) and (2) satisfying one of the following boundary conditions (see Figure 1):

$$
\text { for } \xi=\xi_{1} \text { : }
$$

(a) $\bar{u}=f_{11}$,

$\bar{v}=f_{12}$,

$\bar{p}_{1}=f_{13}$,

$\bar{p}_{2}=f_{14}$, or

(b) $\frac{h_{0}^{2}}{\mu} \sigma_{\xi \xi}=g_{11}$,

$\frac{h_{0}^{2}}{\mu} \tau_{\xi \eta}=g_{12}$

$\bar{p}_{1}=f_{13}$,

$\bar{p}_{2}=f_{14}$, or

(c) $\bar{u}=f_{11}$,

$\bar{v}=f_{12}$,

$$
\frac{\partial \bar{p}_{1}}{\partial \eta}=f_{23},
$$

$\frac{\partial \bar{p}_{2}}{\partial \eta}=f_{24}$, or

(d) $\frac{h_{0}^{2}}{\mu} \sigma_{\xi \xi}=g_{11}$,

$\frac{h_{0}^{2}}{\mu} \tau_{\xi \eta}=g_{12}$,

$\frac{\partial \bar{p}_{1}}{\partial \eta}=f_{23}$,

$\frac{\partial \bar{p}_{2}}{\partial \eta}=f_{24}$.

Here $f_{j k}$ and $g_{j k}$ are given functions. 




FIgURE 2: Semiellipse.

Note that when stress components $\left(h_{0}^{2} / \mu\right) \sigma_{\xi \xi}$ and $\left(h_{0}^{2} / \mu\right) \tau_{\xi \eta}$ are given on boundary $\xi=\xi_{1}$, then the BVP is solvable if the principal vector and the principal moment of external stresses are equal to zero.

Clearly, BVPs for (2) can be investigated separately (Problem $B)$. Then, by admitting $p_{1}(\mathbf{x})$ and $p_{2}(\mathbf{x})$ as known, we can study BVPs for system (1) with respect to $\mathbf{u}(\mathbf{x})$ (Problem A). By combining the obtained results, we arrive at the explicit solutions of BVPs for systems (1)-(2) and (21).

Problem A. First we state the BVPs for the semiellipse, when the conditions of uninterrupted continuation of solutions (i.e., the conditions of symmetry or antisymmetry) are given on $\eta=0, \eta=\pi$, and $\xi=0$ [22]. Thus, we obtain the solutions of BVPs for the ellipse.

Now let us formulate the boundary value problems for semiellipse as follows: find in domain $\Omega_{1}=\left\{0 \leq \xi<\xi_{1}, 0 \leq\right.$ $\eta<\pi\}$ a regular solution $\widetilde{\mathbf{v}}(\bar{u}, \bar{v}) \in C^{2}(\Omega)$ of (19) satisfying the following boundary conditions (see Figure 2):

$$
\begin{aligned}
& \text { for } \eta=0 \text { : } \\
& \text { (a) } \bar{v}=0 \text {, } \\
& \tau_{\xi \eta}=0 \Longleftrightarrow \\
& \bar{v}=0, \\
& \frac{\partial \bar{u}}{\partial \eta}=0 \text { or } \\
& \sigma_{\eta \eta}=0 \Longleftrightarrow \\
& \bar{u}=0 \text {, } \\
& \frac{\partial \bar{v}}{\partial \eta}=0,
\end{aligned}
$$

for $\eta=\pi$ :

$$
\text { (a) } \begin{aligned}
\bar{v} & =0, \\
\tau_{\xi \eta} & =0 \Longleftrightarrow \\
\bar{v} & =0, \\
\frac{\partial \bar{u}}{\partial \eta} & =0 \text { or }
\end{aligned}
$$

(b) $\bar{u}=0$,

$$
\begin{aligned}
& \sigma_{\eta \eta}=0 \Longleftrightarrow \\
& \bar{u}=0, \\
& \frac{\partial \bar{v}}{\partial \eta}=0,
\end{aligned}
$$

for $\xi=0$ :

(a) $u=0$

$$
\begin{aligned}
& \tau_{\xi \eta}=0 \Longleftrightarrow \\
& u=0, \\
& \frac{\partial v}{\partial \xi}=0 \text { or } \\
& v=0, \\
& \sigma_{\xi \xi}=0 \Longleftrightarrow \\
& v=0, \\
& \frac{\partial u}{\partial \xi}=0,
\end{aligned}
$$$$
\text { (b) } v=0 \text {, }
$$

for $\xi=\xi_{1}$ :

$$
\begin{aligned}
& \text { (a) } \bar{u}=f_{11}, \\
& \bar{v}=f_{12} \text { or } \\
& \text { (b) } \frac{h_{0}^{2}}{\mu} \sigma_{\xi \xi}=g_{11}, \\
& \frac{h_{0}^{2}}{\mu} \tau_{\xi \eta}=g_{12} .
\end{aligned}
$$

The solutions of (19) and (22)-(25) are represented by means of two harmonic functions $\varphi_{1}(\xi, \eta)$ and $\varphi_{2}(\xi, \eta)$. Consider

$$
\begin{aligned}
\bar{u} & =\left[\sinh ^{2} \xi_{1} \operatorname{coth} \xi\left(\frac{\partial \varphi_{1}}{\partial \eta}+\frac{\partial \varphi_{2}}{\partial \xi}\right)+(\kappa-1) \varphi_{2}\right] \sinh \xi \\
& \cdot \cos \eta-\left[\cosh ^{2} \xi_{1} \tanh \xi\left(\frac{\partial \varphi_{1}}{\partial \xi}-\frac{\partial \varphi_{2}}{\partial \eta}\right)\right. \\
& \left.-(\kappa-1) \varphi_{1}\right] \cosh \xi \sin \eta, \\
\bar{v}= & -\left[\cosh ^{2} \xi_{1} \tanh \xi\left(\frac{\partial \varphi_{1}}{\partial \eta}+\frac{\partial \varphi_{2}}{\partial \xi}\right)+(\kappa-1) \varphi_{2}\right] \\
& \cdot \cosh \xi \sin \eta-\left[\sinh ^{2} \xi_{1} \operatorname{coth} \xi\left(\frac{\partial \varphi_{1}}{\partial \xi}-\frac{\partial \varphi_{2}}{\partial \eta}\right)\right. \\
& \left.-(\kappa-1) \varphi_{1}\right] \sinh \xi \cos \eta,
\end{aligned}
$$




$$
\begin{aligned}
D & =\frac{\kappa \mu}{\cosh (2 \xi)-\cos (2 \eta)}\left[\left(\frac{\partial \varphi_{1}}{\partial \xi}-\frac{\partial \varphi_{2}}{\partial \eta}\right) \cosh \xi \sin \eta\right. \\
& \left.+\left(\frac{\partial \varphi_{1}}{\partial \eta}+\frac{\partial \varphi_{2}}{\partial \xi}\right) \sinh \xi \cos \eta\right], \\
K & =\frac{\kappa \mu}{\cosh (2 \xi)-\cos (2 \eta)}\left[\left(\frac{\partial \varphi_{1}}{\partial \xi}-\frac{\partial \varphi_{2}}{\partial \eta}\right) \sinh \xi \cos \eta\right. \\
& \left.-\left(\frac{\partial \varphi_{1}}{\partial \eta}+\frac{\partial \varphi_{2}}{\partial \xi}\right) \cosh \xi \sin \eta\right] .
\end{aligned}
$$

The components of stress vector (17) can be written as

$$
\begin{aligned}
& \frac{h_{0}^{2}}{\mu} \sigma_{\xi \xi}=\left[2 \sinh ^{2} \xi_{1} \frac{\partial}{\partial \eta}\left(\frac{\partial \varphi_{1}}{\partial \xi}-\frac{\partial \varphi_{2}}{\partial \eta}\right) \cosh \xi\right. \\
& \left.-\left((\kappa-2) \frac{\partial \varphi_{1}}{\partial \eta}-\kappa \frac{\partial \varphi_{2}}{\partial \xi}\right) \sinh \xi\right] \cos \eta+[2 \\
& \cdot \cosh ^{2} \xi_{1} \frac{\partial}{\partial \eta}\left(\frac{\partial \varphi_{1}}{\partial \eta}+\frac{\partial \varphi_{2}}{\partial \xi}\right) \sinh \xi \\
& \left.+\left(\kappa \frac{\partial \varphi_{1}}{\partial \xi}+(\kappa-2) \frac{\partial \varphi_{2}}{\partial \eta}\right) \cosh \xi\right] \sin \eta \\
& -\frac{4 \sinh \left(\xi_{1}+\xi\right) \sinh \left(\xi_{1}-\xi\right)}{\cosh (2 \xi)-\cos (2 \eta)}\left\{\left(\frac{\partial \varphi_{1}}{\partial \xi}-\frac{\partial \varphi_{2}}{\partial \eta}\right)\right. \\
& \left.\cdot \cosh \xi \sin \eta+\left(\frac{\partial \varphi_{1}}{\partial \eta}+\frac{\partial \varphi_{2}}{\partial \xi}\right) \sinh \xi \cos \eta\right\}, \\
& \frac{h_{0}^{2}}{\mu} \sigma_{\eta \eta}=-\left[2 \sinh ^{2} \xi_{1} \frac{\partial}{\partial \eta}\left(\frac{\partial \varphi_{1}}{\partial \xi}-\frac{\partial \varphi_{2}}{\partial \eta}\right) \cosh \xi\right. \\
& \left.-\left(\kappa \frac{\partial \varphi_{1}}{\partial \eta}-(\kappa-2) \frac{\partial \varphi_{2}}{\partial \xi}\right) \sinh \xi\right] \cos \eta-[2 \\
& \cdot \cosh ^{2} \xi_{1} \frac{\partial}{\partial \eta}\left(\frac{\partial \varphi_{1}}{\partial \eta}+\frac{\partial \varphi_{2}}{\partial \xi}\right) \sinh \xi \\
& \left.+\left((\kappa-2) \frac{\partial \varphi_{1}}{\partial \xi}+\kappa \frac{\partial \varphi_{2}}{\partial \eta}\right) \cosh \xi\right] \sin \eta \\
& +\frac{4 \sinh \left(\xi_{1}+\xi\right) \sinh \left(\xi_{1}-\xi\right)}{\cosh (2 \xi)-\cos (2 \eta)}\left\{\left(\frac{\partial \varphi_{1}}{\partial \xi}-\frac{\partial \varphi_{2}}{\partial \eta}\right)\right. \\
& \left.\cdot \cosh \xi \sin \eta+\left(\frac{\partial \varphi_{1}}{\partial \eta}+\frac{\partial \varphi_{2}}{\partial \xi}\right) \sinh \xi \cos \eta\right\}, \\
& \frac{h_{0}^{2}}{\mu} \tau_{\xi \eta}=-\left[2 \cosh ^{2} \xi_{1} \frac{\partial}{\partial \eta}\left(\frac{\partial \varphi_{1}}{\partial \xi}-\frac{\partial \varphi_{2}}{\partial \eta}\right) \sinh \xi\right. \\
& \left.-\left((\kappa-2) \frac{\partial \varphi_{1}}{\partial \eta}-\kappa \frac{\partial \varphi_{2}}{\partial \xi}\right) \cosh \xi\right] \sin \eta+[2 \\
& \cdot \sinh ^{2} \xi_{1} \frac{\partial}{\partial \eta}\left(\frac{\partial \varphi_{1}}{\partial \eta}+\frac{\partial \varphi_{2}}{\partial \xi}\right) \cosh \xi
\end{aligned}
$$

$$
\begin{aligned}
& \left.+\left(\kappa \frac{\partial \varphi_{1}}{\partial \xi}+(\kappa-2) \frac{\partial \varphi_{2}}{\partial \eta}\right) \sinh \xi\right] \cos \eta \\
& +\frac{4 \sinh \left(\xi_{1}+\xi\right) \sinh \left(\xi_{1}-\xi\right)}{\cosh (2 \xi)-\cos (2 \eta)}\left\{\left(\frac{\partial \varphi_{1}}{\partial \xi}-\frac{\partial \varphi_{2}}{\partial \eta}\right)\right. \\
& \left.\cdot \sinh \xi \cos \eta-\left(\frac{\partial \varphi_{1}}{\partial \eta}+\frac{\partial \varphi_{2}}{\partial \xi}\right) \cosh \xi \sin \eta\right\} .
\end{aligned}
$$

Let us rewrite conditions (25) when $\xi=\xi_{1}$ in the following equivalent form:

(a) $\frac{2}{h_{0}^{2}}\left(\cosh \xi_{1} \sin \eta \bar{u}+\sinh \xi_{1} \cos \eta \bar{v}\right)$

$$
\begin{aligned}
& =-\frac{1}{2} \sinh \left(2 \xi_{1}\right)\left(\frac{\partial \varphi_{1}}{\partial \xi}-\frac{\partial \varphi_{2}}{\partial \eta}\right)+(\kappa-1) \varphi_{1}, \\
& \frac{2}{h_{0}^{2}}\left(\sinh \xi_{1} \cos \eta \bar{u}-\cosh \xi_{1} \sin \eta \bar{v}\right) \\
& =\frac{1}{2} \sinh \left(2 \xi_{1}\right)\left(\frac{\partial \varphi_{1}}{\partial \eta}+\frac{\partial \varphi_{2}}{\partial \xi}\right)+(\kappa-1) \varphi_{2} \text { or }
\end{aligned}
$$

(b) $\frac{2}{\mu}\left(\cosh \xi_{1} \sin \eta \sigma_{\xi \xi}+\sinh \xi_{1} \cos \eta \tau_{\xi \eta}\right)$

$$
\begin{aligned}
= & \sinh \left(2 \xi_{1}\right) \frac{\partial}{\partial \eta}\left(\frac{\partial \varphi_{1}}{\partial \eta}+\frac{\partial \varphi_{2}}{\partial \xi}\right)+\kappa \frac{\partial \varphi_{1}}{\partial \xi} \\
& +(\kappa-2) \frac{\partial \varphi_{2}}{\partial \eta}
\end{aligned}
$$$$
\frac{2}{\mu}\left(\sinh \xi_{1} \cos \eta \sigma_{\xi \xi}-\cosh \xi_{1} \sin \eta \tau_{\xi \eta}\right)
$$$$
=\sinh \left(2 \xi_{1}\right) \frac{\partial}{\partial \eta}\left(\frac{\partial \varphi_{1}}{\partial \xi}-\frac{\partial \varphi_{2}}{\partial \eta}\right)-(\kappa-2) \frac{\partial \varphi_{1}}{\partial \eta}
$$$$
+\kappa \frac{\partial \varphi_{2}}{\partial \xi}
$$

In (28), functions $\varphi_{1}$ and $\varphi_{2}$ are harmonic functions, which, by using the method of separation of variables $[23,24]$, can be presented as follows:

$$
\varphi_{i}=\sum_{n=1}^{\infty} \varphi_{i n}, \quad i=1,2,
$$

where

$$
\begin{aligned}
& \varphi_{1 n}=B_{1 n} \frac{\sinh (n \xi)}{\cosh \left(n \xi_{1}\right)} \sin (n \eta), \\
& \varphi_{2 n}=B_{2 n} \frac{\cosh (n \xi)}{\cosh \left(n \xi_{1}\right)} \cos (n \eta) \text { or } \\
& \varphi_{1 n}=B_{1 n} \frac{\cosh (n \xi)}{\cosh \left(n \xi_{1}\right)} \cos (n \eta), \\
& \varphi_{2 n}=B_{2 n} \frac{\sinh (n \xi)}{\cosh \left(n \xi_{1}\right)} \sin (n \eta) .
\end{aligned}
$$




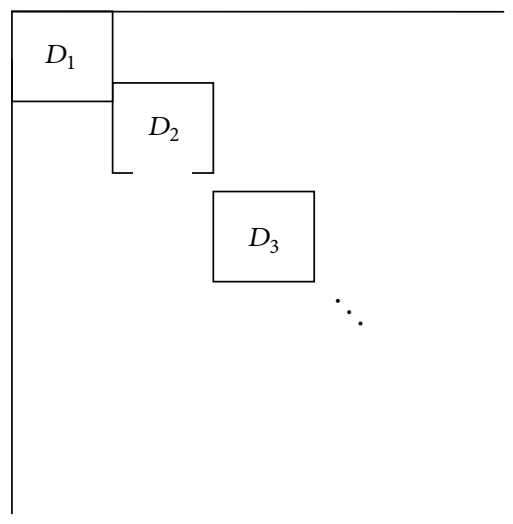

FIGURE 3: Diagram of the main matrix of the algebraic equation system.

When $n=0$, then $\varphi_{10}=B_{10}+a_{02} \xi+a_{03} \eta+a_{04} \xi \eta$ and $\varphi_{20}=B_{20}+b_{02} \xi+b_{03} \eta+b_{04} \xi \eta$, where $B_{10}, a_{02}, \ldots, b_{04}$ are constants. From the condition of limitation of $\operatorname{grad} \varphi_{i 0}=$ $(1 / h)\left(\partial \varphi_{i 0} / \partial \xi+\partial \varphi_{i 0} / \partial \alpha\right)$ in the focuses of ellipse and condition of periodicity of functions $\varphi_{i 0}$ along $\eta$ coordinate, there ensues the following: $a_{0 j}=0, b_{0 j}=0, j=2,3,4$. Therefore, $\varphi_{10}=0$ and $\varphi_{20}=A_{20}$ or $\varphi_{10}=A_{10}$, and $\varphi_{20}=0$. When on boundary $\xi=\xi_{0}$ the values of the stress vector are given, then the rigid displacements of the ellipse are defined by the equalities. $\varphi_{10}=0$ and $\varphi_{20}=A_{20}$ and $\varphi_{10}=A_{10}$, and $\varphi_{20}=0$.

By substituting (22)-(24) and (29) into (28), for determining unknown $B_{1 n}$ and $B_{2 n}$, we get the following infinite system of algebraic equations, whose main matrix has a block-diagonal form (see Figure 3).

The dimension of all matrices $D_{i}(i=1,2, \ldots)$ is $2 \times 2$ and $\operatorname{det} D_{i} \neq 0$. When $i \rightarrow \infty$, $\operatorname{det} D_{i} \rightarrow M$, where $M \neq 0$.

Thus, the system is uniquely solvable. Let us substitute the obtained values, $B_{1 n}$ and $B_{2 n}$, into $\varphi_{1}$ and $\varphi_{2}$. As a result, we obtain the vector $\widetilde{\mathbf{v}}(\bar{u}, \bar{v})$.

Problem $B$. Find regular solutions $p_{1}$ and $p_{2}$ to (2) in domain $\Omega=\left\{0 \leq \xi<\xi_{1}, 0 \leq \eta<2 \pi\right\}$ satisfying one of the following boundary conditions (see Figure 1):

when $\xi=\xi_{1}$ :

$$
\text { (a) } \begin{aligned}
p_{1} & =f_{13}, \\
p_{2} & =f_{14} \text { or } \\
\text { (b) } \frac{\partial p_{1}}{\partial \eta} & =f_{23}, \\
\frac{\partial p_{2}}{\partial \eta} & =f_{24} .
\end{aligned}
$$

The solution of (2) is (18), where function $\overline{\phi_{1}}$ is a solution of (16). The solution of (16) by applying the method of separation of variables is reduced to solutions of the Mathieu differential equation or modified Mathieu differential equations (see Appendix B) [25]. These solutions are known as Mathieu functions. Thus, $\overline{\phi_{1}}(\xi, \eta)=R(\xi) \cdot \Phi_{2}(\eta)$, where $\Phi_{2}(\eta)$ is the solution of Mathieu differential equation $d^{2} \Phi_{2}(\eta)(\eta) / d \eta^{2}+$ $[a-2 q \cos (2 \eta)] \Phi_{2}(\eta)(\eta)=0$ (see Appendix C), and $R$ is the solution of modified Mathieu differential equations $d^{2} R(\xi) / d \xi^{2}-[a-2 q \cosh (2 \xi)] R(\xi)=0$ (see Appendix D), where $a:=c+(1 / 2) \lambda_{1}^{2}, q:=(1 / 4) \lambda_{1}^{2}, c=$ const is an arbitrary constant.

Let us assume that harmonic function $\bar{\phi}$ from (18) is represented in the form of series:

$$
\begin{aligned}
\bar{\phi}= & \sum_{n=0}^{\infty} C_{n} \cosh (n \xi) \cos (n \eta) \\
& +\sum_{n=1}^{\infty} D_{n} \sinh (n \xi) \sin (n \eta) .
\end{aligned}
$$

Taking into account the boundary conditions (31), to determine unknown coefficients $C_{n}$ and $D_{n}$, we obtain the system of infinite algebraic equations, whose main matrix has a block-diagonal form (Figure 3). By solving this system, we find $\bar{\phi}(\xi, \eta)$.

For determining $\bar{\varphi}_{0}$ from (17), let us consider the particular cases: keeping in mind the homogeneous boundary conditions, we get $C_{n}=0$ or $D_{n}=0$. Let us assume that $D_{n}=0$.

Substituting (32) in (17), we obtain the following equation:

$$
\begin{aligned}
\frac{\partial^{2} \bar{\varphi}_{0}}{\partial \xi^{2}} & +\frac{\partial^{2} \bar{\varphi}_{0}}{\partial \eta^{2}} \\
= & \sum_{k=0}^{\infty} C_{k} \cosh (k \xi) \cos (k \eta)(\cosh (2 \xi)-\cos (2 \eta)) \\
= & \sum_{n=0}^{\infty} E_{n} \cosh (n \xi) \cos (n \eta) .
\end{aligned}
$$

The solution of (33) is sought in the following form:

$$
\bar{\varphi}_{0}=\bar{\varphi}_{0 g}+\bar{\varphi}_{0 p}
$$

where $\bar{\varphi}_{0 g}$ is a harmonic function and it can be obtained in the same way as $\bar{\phi}$. Thus, we have

$$
\begin{aligned}
\Delta \bar{\varphi}_{0 g} & =0 \\
\bar{\varphi}_{0 g} & =\sum_{n=0}^{\infty} T_{n} \cosh (n \xi) \cos (n \eta) .
\end{aligned}
$$

$\bar{\varphi}_{0 p}$ is the particular solution of the following equation:

$$
\frac{\partial^{2} \bar{\varphi}_{0 p}}{\partial \xi^{2}}+\frac{\partial^{2} \bar{\varphi}_{0 p}}{\partial \eta^{2}}=\sum_{n=0}^{\infty} E_{n} \cosh (n \xi) \cos (n \eta)
$$

The solution of (36) is sought in the form

$$
\bar{\varphi}_{0 p}=\sum_{n=0}^{\infty} Q_{n} \cosh (n \xi) \cos (n \eta)
$$


Substituting (37) in (36), we obtain the relations between $Q_{n}$ and $E_{n}$ and hence it follows that

$$
\begin{aligned}
\bar{\varphi}_{0} & =\sum_{n=0}^{\infty} T_{n} \cosh (n \xi) \cos (n \eta) \\
& +\sum_{n=0}^{\infty} Q_{n} \cosh (n \xi) \cos (n \eta)
\end{aligned}
$$

or

$$
\bar{\varphi}_{0}=\sum_{n=0}^{\infty} M_{n} \cosh (n \xi) \cos (n \eta),
$$

where $M_{n}=T_{n}+Q_{n}$.

Quite similarly, we obtain the solution, when $C_{n}=0$. By combining the obtained results, we obtain an explicit solution of (17).

Remark 1. When we have the nonhomogeneous boundary conditions, then the coefficients in (32) are different from zero, $C_{n} \neq 0, D_{n} \neq 0$, and function $\bar{\varphi}_{0}$ will be obtained by above-mentioned method for each series, separately.

By substituting $\bar{\varphi}_{0}$ and $\bar{\phi}_{1}$ in (14), we obtain function $\widetilde{\mathbf{v}}_{0}$. Finally, from (13), we obtain displacement $\widetilde{\mathbf{u}}$ in $\Omega$ at arbitrary point. Stress components are defined from (20).

\section{Conclusions}

The main results of this work can be formulated as follows:

(1) The system of equations of the linear equilibrium theory of elasticity for solids with double porosity structure is written in terms of elliptic coordinates.

(2) The problems are reduced to the solvability of two types of problem. The first one is similar to the BVPs for the equations of classical elasticity of isotropic bodies, while the second one is the BVPs for the equations of the porous and fissure fluid pressures.

(3) Analytical (exact) solutions are obtained for 2D BVPs for the ellipse with double porosity structure.

By using the above-mentioned method, the following is possible:

(4) It is possible to construct explicitly the solutions of basic BVPs for systems (1) and (2) for simple cases of 2D domains (circle, plane with circular hole.) in the form of absolutely and uniformly convergent series that are useful in the engineering practice.

(5) It is possible to obtain numerical solutions of the boundary value problems.

(6) It is possible to construct explicitly the solutions of basic BVPs of the systems of equations in the modern linear theories of elasticity, thermoelasticity, and poroelasticity for materials with microstructures and for elastic materials with double porosity for a circle, and so forth.

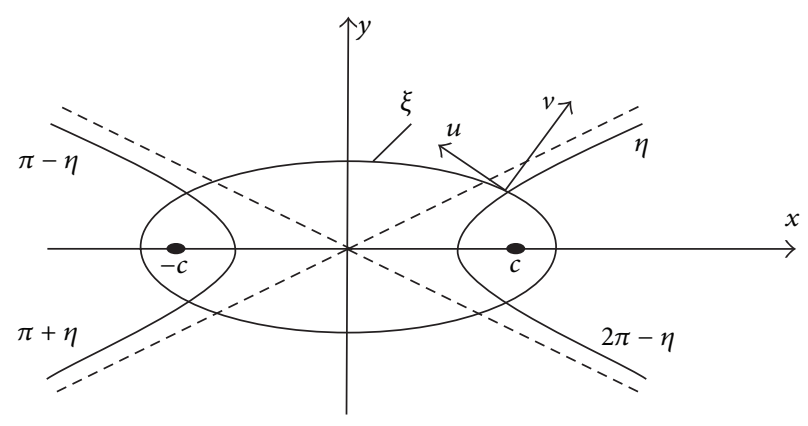

FIgURE 4: Elliptic coordinate system.

(7) In practice, such BVPs are quite common in many areas of science. The potential users of the obtained results will be the scientists and engineers working on the problems of solid mechanics, micromechanics and nanomechanics, mechanics of materials, engineering mechanics, engineering medicine, biomechanics, engineering geology, geomechanics, hydroengineering, applied and computing mechanics, and applied mathematics.

\section{Appendix}

\section{A. Some Basic Formulas in Elliptic Coordinates}

If $x, y(-\infty<x<+\infty,-\infty<y<+\infty)$ are Cartesian coordinates and $\xi, \eta$ are elliptic coordinates $(0 \leq \xi<\infty, 0 \leq$ $\eta<2 \pi)$, then $x=c \cosh \xi \cos \eta, y=c \sinh \xi \sin \eta$, where $c$ is scale factor (Figure 4) [26].

The coordinate curves are ellipses and hyperbolas:

$$
\begin{aligned}
& \frac{x^{2}}{c^{2} \cosh ^{2} \xi_{0}}+\frac{y^{2}}{c^{2} \sinh ^{2} \xi_{0}}=1, \quad \xi_{0}=\text { const } \neq 0, \\
& \frac{x^{2}}{c^{2} \cos ^{2} \eta_{0}}-\frac{y^{2}}{c^{2} \sin ^{2} \eta_{0}}=1, \\
& \eta_{0}=\text { const } \neq 0, \frac{\pi}{2}, \pi, \frac{3}{2} \pi .
\end{aligned}
$$

$h:=h_{\xi}=h_{\eta}=(c / \sqrt{2}) \sqrt{\cosh (2 \xi)-\cos (2 \eta)}=$ $c \sqrt{\sinh ^{2} \xi+\sin ^{2} \eta}$ are metric coefficients (Lamé coefficients). form

The Laplacian operator in the elliptic coordinates has the

$$
\Delta f=\frac{1}{h^{2}}\left(\frac{\partial^{2} f}{\partial \xi^{2}}+\frac{\partial^{2} f}{\partial \eta^{2}}\right) .
$$

The operator grad has the form

$$
\nabla f:=\operatorname{grad}(f)=\frac{1}{h}\left(\frac{\partial f}{\partial \xi} \vec{e}_{\xi}+\frac{\partial f}{\partial \eta} \vec{e}_{\eta}\right),
$$

where $f=f(\xi, \eta)$. 
The Helmholtz equation can be written as

$$
\frac{1}{h^{2}}\left(\frac{\partial^{2} f}{\partial \xi^{2}}+\frac{\partial^{2} f}{\partial \eta^{2}}\right)+\lambda_{1}^{2} f=0
$$

where $f=f(\xi, \eta)$,

and biharmonic equation has the form

$$
\begin{aligned}
\Delta \Delta f & =(\Delta f)^{2}=\left[\frac{1}{h^{2}}\left(\frac{\partial^{2} f}{\partial \xi^{2}}+\frac{\partial^{2} f}{\partial \eta^{2}}\right)\right]^{2} \\
& =\frac{1}{h^{4}}\left(\frac{\partial^{4} f}{\partial \xi^{4}}+2 \frac{\partial^{4} f}{\partial \xi^{2} \partial \eta^{2}}+\frac{\partial^{4} f}{\partial \eta^{4}}\right)=0 .
\end{aligned}
$$

\section{B. Solution of Helmholtz Equation in the Elliptic Coordinates}

Helmholtz equation in the elliptic coordinateshas the following form:

$$
\frac{1}{\sinh ^{2} u+\sin ^{2} v}\left(\frac{\partial^{2} F}{\partial u^{2}}+\frac{\partial^{2} F}{\partial v^{2}}\right)+k^{2} F=0 .
$$

Let us solve this equation by using the method of separation of variables.

Let function $F$ be sought in the form

$$
F(u, v)=U(u) \cdot V(v),
$$

and then Helmholtz equation takes the following form:

$$
\frac{1}{\sinh ^{2} u+\sin ^{2} v}\left(V \frac{d^{2} U}{d u^{2}}+U \frac{d^{2} V}{d v^{2}}\right)+k^{2} U V=0 .
$$

From here, we obtain

$$
\frac{1}{\sinh ^{2} u+\sin ^{2} v}\left(\frac{1}{U} \frac{d^{2} U}{d u^{2}}+\frac{1}{V} \frac{d^{2} V}{d v^{2}}\right)+k^{2}=0 .
$$

Let us rewrite the last equation in the following form:

$$
\left(\frac{1}{U} \frac{\partial^{2} U}{d u^{2}}+k^{2} \sinh ^{2} u\right)+\left(\frac{1}{V} \frac{\partial^{2} V}{d v^{2}}+k^{2} \sin ^{2} v\right)=0 .
$$

From here, we get

$$
\begin{gathered}
\frac{1}{U} \frac{d^{2} U}{d u^{2}}+k^{2} \sinh ^{2} u=c, \\
c+\frac{1}{V} \frac{d^{2} V}{d v^{2}}+k^{2} \sin ^{2} v=0 .
\end{gathered}
$$

Thus,

$$
\begin{gathered}
\frac{d^{2} U}{d u^{2}}-\left(c-k^{2} \sinh ^{2} u\right) U=0 \\
\frac{d^{2} V}{d v^{2}}+\left(c+k^{2} \sin ^{2} v\right) V=0 .
\end{gathered}
$$

Take into account the following identities:

$$
\begin{aligned}
\sinh ^{2} u & =\frac{1}{2}[\cosh (2 u)-1], \\
\sin ^{2} v & =\frac{1}{2}[1-\cos (2 v)] .
\end{aligned}
$$

We obtain

$$
\begin{aligned}
& \frac{d^{2} U}{d u^{2}}-\left\{c-\frac{1}{2} k^{2}[\cosh (2 u)-1]\right\} U=0, \\
& \frac{d^{2} V}{d v^{2}}+\left\{c+\frac{1}{2} k^{2}[1-\cos (2 v)]\right\} V=0 .
\end{aligned}
$$

From here, we get

$$
\begin{gathered}
\frac{\partial^{2} U}{\partial u^{2}}-\left[\left(c+\frac{1}{2} k^{2}\right)-\frac{1}{2} k^{2} \cosh (2 u)\right] U=0 \\
\frac{d^{2} V}{d v^{2}}+\left[\left(c+\frac{1}{2} k^{2}\right)-\frac{1}{2} k^{2} \cos (2 v)\right] V=0 .
\end{gathered}
$$

Let us introduce the notations $a:=c+(1 / 2) k^{2}$ and $q:=$ $(1 / 4) k^{2}$; then we have $d^{2} U / d u^{2}-[a-2 q \cosh (2 u)] U=0$; this is the modification differential equation of Mathieu. Consider $d^{2} V / d v^{2}+[a-2 q \cos (2 v)] V=0$; this is the differential equation of Mathieu.

\section{The Solutions of Differential Equation of Mathieu}

Let us assume that $c=0$; then $a:=\left(\lambda_{1} / \sqrt{2}\right)^{2}$ and $q:=$ $(1 / 4) \lambda_{1}{ }^{2}$. The differential equation of Mathieu takes the form

$$
\frac{\partial^{2} \Phi_{2}(\eta)}{\partial \eta^{2}}+(a-2 q \cos 2 \eta) \Phi_{2}(\eta)=0
$$

The solutions of this equation have the following forms [25]:

(a) When $q>0$,

$$
\begin{aligned}
c e_{2 m}(q, \eta) & =\sum_{n=0}^{\infty} A_{2 n}^{(2 m)}(q) \cos (2 n \eta), \\
s e_{2 m+2}(q, \eta) & =\sum_{n=0}^{\infty} B_{2 n+2}^{(2 m+2)}(q) \sin [(2 n+2) \eta], \\
c e_{2 m+1}(q, \eta) & =\sum_{n=0}^{\infty} A_{2 n+1}^{2 m+1}(q) \cos [(2 n+1) \eta], \\
s e_{2 m+1}(q, \eta) & =\sum_{n=0}^{\infty} B_{2 n+1}^{(2 m+1)}(q) \sin [(2 n+1) \eta] .
\end{aligned}
$$


(b) When $q<0$,

$$
\begin{aligned}
& c e_{2 m}(-q, \eta)=(-1)^{m} \sum_{n=0}^{\infty}(-1)^{n} A_{2 n}^{(2 m)}(q) \cos (2 n \eta), \\
& s e_{2 m+2}(-q, \eta) \\
& \quad=(-1)^{m} \sum_{n=0}^{\infty}(-1)^{n} B_{2 n+2}^{(2 m+2)}(q) \sin [(2 n+2) \eta] \\
& c e_{2 m+1}(-q, \eta) \\
& \quad=(-1)^{m} \sum_{n=0}^{\infty}(-1)^{n} A_{2 n+1}^{(2 m+1)}(q) \cos [(2 n+1) \eta] \\
& s e_{2 m+1}(-q, \eta) \\
& \quad=(-1)^{m} \sum_{n=0}^{\infty}(-1)^{n} B_{2 n+1}^{(2 m+1)}(q) \sin [(2 n+1) \eta]
\end{aligned}
$$

where, for $c e_{2 m}(q, \eta)$,

$$
\begin{aligned}
a A_{0}-q A_{2} & =0, \\
(a-4) A_{2}-q\left(A_{4}+2 A_{0}\right) & =0, \\
\left(a-4 n^{2}\right) A_{2 n}-q\left(A_{2 n+2}+A_{2 n-2}\right) & =0, \quad n \geq 2 ;
\end{aligned}
$$

for $c e_{2 m+1}(q, \eta)$,

$$
\begin{array}{r}
(a-1-q) A_{1}-q A_{3}=0, \\
{\left[a-(2 n+1)^{2}\right] A_{2 n+1}-q\left(A_{2 n+3}+A_{2 n-1}\right)=0,}
\end{array}
$$

$n \geq 1$

for $\operatorname{se}_{2 m+1}(q, \eta)$,

$$
\begin{aligned}
(a-1+q) B_{1}-q B_{3} & =0, \\
{\left[a-(2 n+1)^{2}\right] B_{2 n+1}-q\left(B_{2 n+3}+B_{2 n-1}\right) } & =0,
\end{aligned}
$$

$n \geq 1$;

and, for $s e_{2 m+2}(q, \eta)$,

$$
\begin{aligned}
(a-4) B_{2}-q B_{4} & =0, \\
\left(a-4 n^{2}\right) B_{2 n}-q\left(B_{2 n+2}+B_{2 n-2}\right) & =0, \quad n \geq 2
\end{aligned}
$$

(here $\left.A_{2 n}^{(2 m)} \equiv A_{2 n}, \ldots, B_{2 n+2}^{(2 m+2)} \equiv B_{2 n+2}\right)$.

\section{The Solutions of the Modification Differential Equation of Mathieu}

The modification differential equation of Mathieu has the form

$$
\frac{\partial^{2} R(\xi)}{\partial \eta^{2}}-(a-2 q \cosh 2 \xi) R(\xi)=0 .
$$

The solutions of this equation are as follows [25]:

(a) If $q>0$,

$$
\begin{aligned}
& C e_{2 m}(q, \xi)=\sum_{k=0}^{\infty} A_{2 k} J_{2 k}(2 \sqrt{q} \sinh \xi) \\
& C e_{2 m+1}(q, \xi)=\sum_{k=0}^{\infty}(-1)^{k+1} A_{2 k+1} J_{2 k+1}(2 \sqrt{q}) \cosh \xi \\
& S e_{2 m+1}(q, \xi)=\sum_{k=0}^{\infty} B_{2 k+1} J_{2 k+1}(2 \sqrt{q} \sinh \xi) \\
& S e_{2 m+2}(q, \xi) \\
& \quad=\tanh \xi \sum_{k=0}^{\infty}(-1)^{k} 2 k B_{2 k} J_{2 k}(2 \sqrt{q} \cosh \xi) .
\end{aligned}
$$

(b) If $q<0$, then the argument of Bessel function is $(2 \sqrt{-q}=i 2 \sqrt{q})$.

\section{E. Physical Motivation of Double Porosity Model}

The double porosity model has received a lot of attention from mathematicians and from engineers. In such a model, there are two pore systems with different permeability. There is first a set of isolated porous blocks of low permeability (sometimes called matrix), surrounded by network of high permeability connected porous medium (usually called fractures network). Pores are pervasive in most of the igneous, metamorphic, and sedimentary rocks in the earth's crust. In fact, porosity found in the earth may have many shapes and sizes, but two types of porosity are more important. One is the matrix porosity, and the other is fracture or crack porosity which may occupy very little volume, but fluid flow occurs primarily through the fracture network.

In physical terms, the theory of poroelasticity postulates that when a porous material is subjected to stress, the resulting matrix deformation leads to volumetric changes in the pores. The pores are filled with fluid. The presence of the fluid results in the flow of the pore fluid between regions of higher and lower pore pressure.

The physical process of coupled deformation is governed by the following equations:

(1) The equations of motion $[27,28]$ :

$$
t_{l j, j}=\rho\left(\ddot{u}_{l}-F_{1}\right), \quad l, j=1,2,3,
$$

where $t_{l j}$ is the components of total stress tensor, $\rho>0$ is the reference mass density, and $F$ is the body force per unit mass. We assume that subscripts preceded by a comma denote partial differentiation with respect to the corresponding Cartesian coordinate, repeated indices are summed over the range $(1,2,3)$, and the dot denotes differentiation with respect to $t$ (here $t$ denotes the time variable; $t \geq 0$ ). 
(2) The equations of fluid mass conservation [5]:

$$
\begin{aligned}
& \operatorname{div} \mathbf{v}^{(1)}+\dot{\zeta}_{1}+\beta_{1} \dot{e}_{r r}+v\left(p_{1}-p_{2}\right)=0, \\
& \operatorname{div} \mathbf{v}^{(2)}+\dot{\zeta}_{2}+\beta_{2} \dot{e}_{r r}-v\left(p_{1}-p_{2}\right)=0,
\end{aligned}
$$

where $\mathbf{v}^{(1)}$ and $\mathbf{v}^{(2)}$ are the fluid flux vectors for pores and fissures, respectively.

$e_{l j}$ is the components of strain tensor:

$$
e_{l j}=\frac{1}{2}\left(u_{l, j}+u_{j, l}\right), \quad l, j=1,2,3 .
$$

$\beta_{1}$ and $\beta_{2}$ are the effective stress parameters and $\gamma$ is the internal transport coefficients and corresponds to a fluid transfer rate respecting the intensity of the flow between pores and fissures, $\gamma \geq 0$, and $\varsigma_{1}$ and $\varsigma_{2}$ are the increments of fluid in pores and fissures, respectively, and are defined by

$$
\begin{aligned}
& \varsigma_{1}=\alpha_{1} p_{1}+\alpha_{12} p_{2} \\
& \varsigma_{2}=\alpha_{21} p_{1}+\alpha_{2} p_{2} .
\end{aligned}
$$

$\alpha_{1}$ and $\alpha_{2}$ measure the compressibility of pore and fissure systems, respectively, and $\alpha_{12}$ and $\alpha_{21}$ are the cross-coupling compressibility for fluid flow at the interface between the two pore systems at a microscopic level.

(3) The constitutive equations (extending Terzaghi's effective stress concept to double porosity) $[1,5]$ :

$$
\begin{aligned}
t_{l j}=2 \mu e_{l j}+\lambda e_{r r} \delta_{l j}-\left(\beta_{1} p_{2}+\beta_{1} p_{2}\right) \delta_{l j}, & \\
& l, j=1,2,3 .
\end{aligned}
$$

$\lambda$ and $\mu$ are the Lame constants, and $\delta_{l j}$ is Kronecker's delta.

(4) Darcy's law for material with double porosity [7, 8]:

$$
\begin{aligned}
& \mathbf{v}^{(1)}=-\frac{1}{\mu^{\prime}}\left(\kappa_{1} \operatorname{grad} p_{1}+\kappa_{12} \operatorname{grad} p_{2}\right)-\rho_{1} s^{(1)}, \\
& \mathbf{v}^{(2)}=-\frac{1}{\mu^{\prime}}\left(\kappa_{21} \operatorname{grad} p_{1}+\kappa_{2} \operatorname{grad} p_{2}\right)-\rho_{2} s^{(2)},
\end{aligned}
$$

where $\mu^{\prime}$ is the fluid viscosity, $\kappa_{1}$ and $\kappa_{2}$ are the macroscopic intrinsic permeabilities associated with the matrix and fissure porosity, respectively, and $\kappa_{12}$ and $\kappa_{21}$ are the cross-coupling compressibilities for fluid flow at the interface between the matrix and fissure phases; $\rho_{1}, s^{(1)}$ and $\rho_{2}, s^{(2)}$ are the densities of fluid and the external forces for the pore and fissure phases, respectively.

Substituting (E.3)-(E.6) into (E.1) and (E.2), assuming that $\mathbf{u}=\left(u_{1}, u_{2}\right), p_{j}=p_{j}\left(x_{1}, x_{2}\right), u_{j}=u_{j}\left(x_{1}, x_{2}\right), \rho=0, \rho_{1}=$ $0, s^{(j)}=0$ and $F_{j}=0$, we obtain the system of homogeneous equations of motion in the coupled linear theory of elasticity for solids with double porosity structure (see (1) and (2)).

\section{Competing Interests}

The authors declare that they have no competing interests.

\section{References}

[1] R. K. Wilson and E. C. Aifantis, "On the theory of consolidation with double porosity," International Journal of Engineering Science, vol. 20, no. 9, pp. 1009-1035, 1982.

[2] D. E. Beskos and E. C. Aifantis, "On the theory of consolidation with double porosity-II," International Journal of Engineering Science, vol. 24, no. 11, pp. 1697-1716, 1986.

[3] M. Y. Khaled, D. E. Beskos, and E. C. Aifantis, "On the theory of consolidation with double porosity-III: a finite element formulation," International Journal for Numerical and Analytical Methods in Geomechanics, vol. 8, no. 2, pp. 101-123, 1984.

[4] R. De Boer, Theory of Porous Media: Highlights in Historical Development and Current State, Springer, Berlin, Germany, 2000.

[5] N. Khalili and S. Valliappan, "Unified theory of flow and deformation in double porous media," European Journal of Mechanics, A/Solids, vol. 15, no. 2, pp. 321-336, 1996.

[6] N. Khalili, "Coupling effects in double porosity media with deformable matrix," Geophysical Research Letters, vol. 30, no. 22, 2003.

[7] J. G. Berryman and H. F. Wang, "The elastic coefficients of double-porosity models for fluid transport in jointed rock," Journal of Geophysical Research, vol. 100, no. 12, pp. 24611-24627, 1995.

[8] J. G. Berryman and H. F. Wang, "Elastic wave propagation and attenuation in a double-porosity dual-permeability medium," International Journal of Rock Mechanics and Mining Sciences, vol. 37, no. 1-2, pp. 63-78, 2000.

[9] M. Svanadze and S. De Cicco, "Fundamental solutions in the full coupled theory of elasticity for solids with double porosity," Archives of Mechanics, vol. 65, no. 5, pp. 367-390, 2013.

[10] M. Svanadze, "Fundamental solution in the theory of consolidation with double porosity," Journal of the Mechanical Behavior of Materials, vol. 16, pp. 123-130, 2005.

[11] I. Tsagareli and L. Bitsadze, "Explicit solution of one boundary value problem in the full coupled theory of elasticity for solids with double porosity," Acta Mechanica, vol. 226, no. 5, pp. 14091418, 2015.

[12] M. Basheleishvili and L. Bitsadze, "Explicit solutions of the boundary value problems of the theory of consolidation with double porosity for the half-plane," Georgian Mathematical Journal, vol. 19, no. 1, pp. 41-48, 2012.

[13] L. Bitsadze and I. Tsagareli, "The solution of the Dirichlet BVP in the fully coupled theory of elasticity for spherical layer with double porosity," Meccanica, vol. 51, no. 6, pp. 1457-1463, 2016.

[14] N. Khalili and A. P. S. Selvadurai, "A fully coupled constitutive model for thermo-hydro-mechanical analysis in elastic media with double porosity," Geophysical Research Letters, vol. 30, no. 24, pp. 7-5, 2003.

[15] N. Khalili and A. P. S. Selvadurai, "On the constitutive modelling of thermo-hydro-mechanical coupling in elastic media with double porosity," Elsevier Geo-Engineering Book Series, vol. 2, pp. 559-564, 2004.

[16] B. Straughan, "Stability and uniqueness in double porosity elasticity," International Journal of Engineering Science, vol. 65, pp. 1-8, 2013.

[17] L. Bitsadze and I. Tsagareli, "Solutions of BVPs in the fully coupled theory of elasticity for the space with double porosity and spherical cavity," Mathematical Methods in the Applied Sciences, vol. 39, no. 8, pp. 2136-2145, 2016. 
[18] W. Nowacki, Theory of Elasticity, Translated from Polish B. E. Pobedri, Mir, Moscow, Russia, 1975 (Russian).

[19] A. F. Ulitko, Method Own Vector Functions in Spatial Problems of Elasticity, Naukova Dumka, Kyiv, Ukraine, 1979.

[20] N. G. Khomasuridze, "Elastic equilibrium of multi-layer plates on classical and moment theories," in Proceedings of the AllUnion Conference-Seminar in Tbilisi on the Theory of Numerical Method of Calculation of Plates and Shells, vol. 2, pp. 346-366, 1984.

[21] N. Khomasuridze and N. Zirakashvili, "Some two dimensional elastic equilibrium problems of elliptic bodies," Proceedings of $I$. Vekua Institute of Applied Mathematics, vol. 49, pp. 39-48, 1999.

[22] N. G. Khomasuridze, "The symmetry principle in continuum mechanics," Journal of Applied Mathematics and Mechanics, vol. 71, no. 1, pp. 20-29, 2007.

[23] J. W. Brown and R. V. Churchill, Fourier Series and Boundary Value Problems, McGraw-Hill, New York, NY, USA, 5th edition, 1993.

[24] A. V. Bitsadze, Equations of Mathematical Physics, Mir Publishers, 1980.

[25] E. Jahnke, F. Emde, and F. Lesh, Special Functions. Formulas, Graphs, Tables, Izdat. Nauka, Moscow, Russia, 1977 (Russian).

[26] A. F. Bermant, Mapping Linear Coordinates. Transformation Green's Formulas, Fizmatgiz, Moscow, Russia, 1958 (Russian).

[27] M. A. Biot, "Mechanics of deformation and acoustic propagation in porous media," Journal of Applied Physics, vol. 33, pp. 1482-1498, 1962.

[28] M. A. Biot, "Theory of finite deformations of pourous solids," Indiana University Mathematics Journal, vol. 21, pp. 597-620, 1972. 


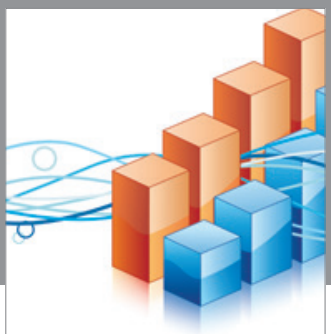

Advances in

Operations Research

vatem alat4

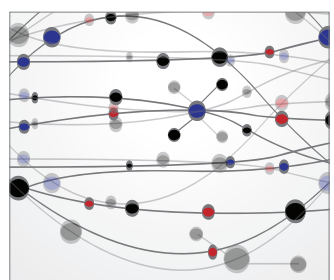

\section{The Scientific} World Journal
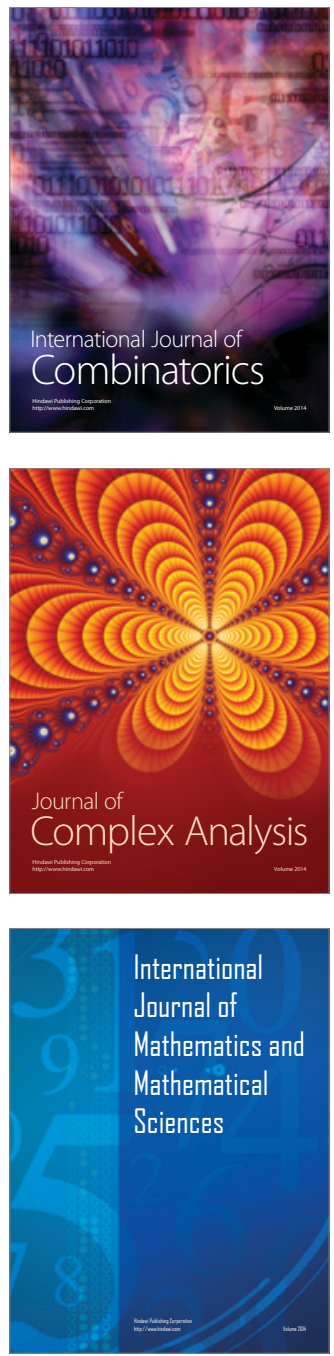
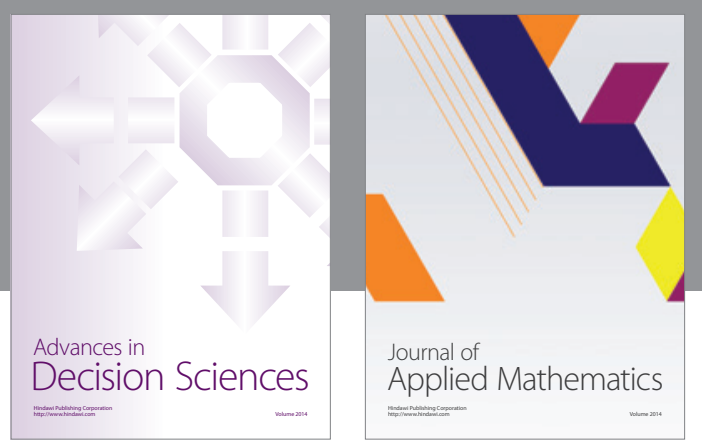

Algebra

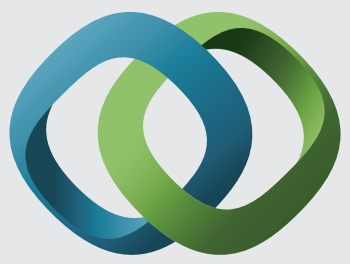

\section{Hindawi}

Submit your manuscripts at

http://www.hindawi.com
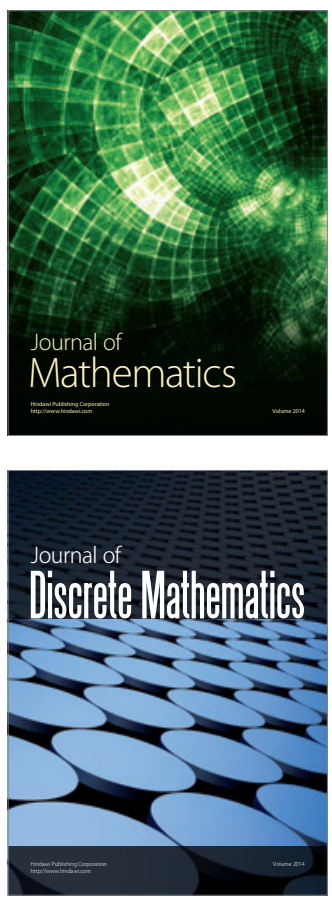

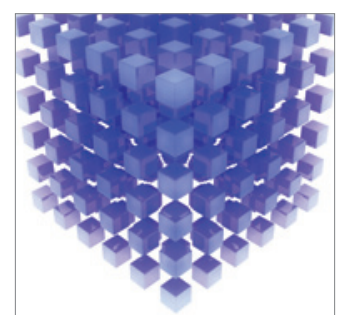

Mathematical Problems in Engineering
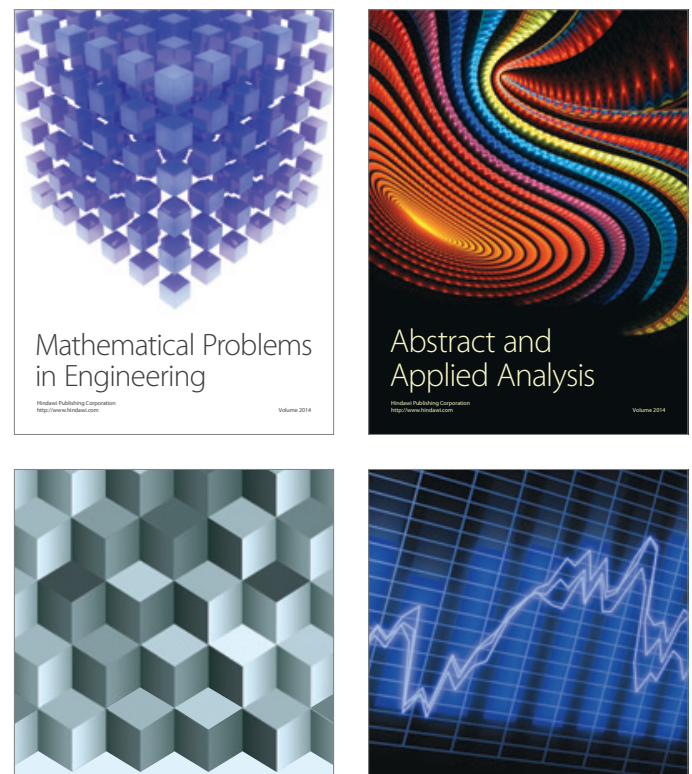

Journal of

Function Spaces

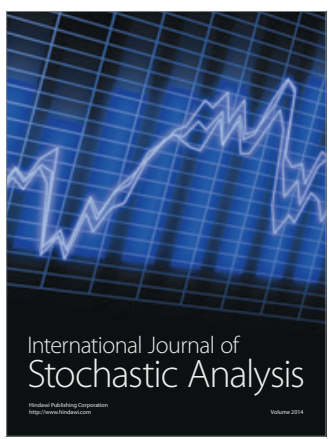

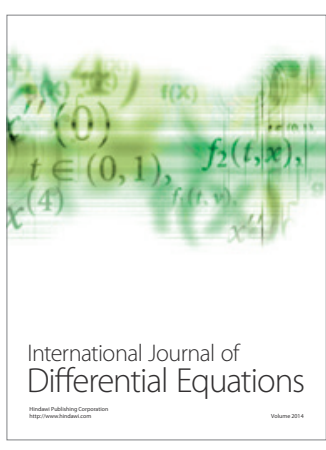
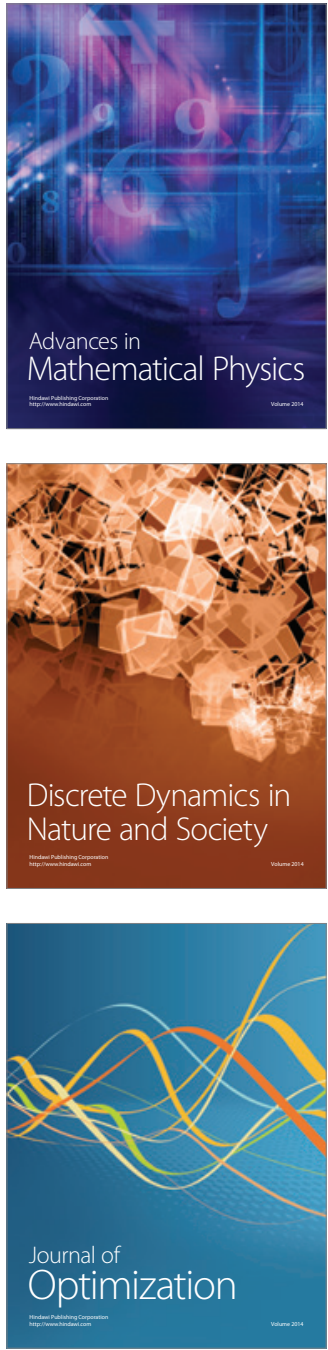